\title{
Alocação de Banda de Guarda Adaptativa Utilizando Redes Neurais Multi Layer Perceptron em Redes Ópticas Elásticas
}

\author{
Neclyeux Monteiro ${ }^{1}$, Wilson Junior ${ }^{1}$, Alexandre Fontinele ${ }^{2}$, \\ Divanilson R. Campelo ${ }^{2}$, Anselmo Paiva ${ }^{3}$, Ricardo Rabelo ${ }^{1}$ e André Soares ${ }^{1}$ \\ ${ }^{1}$ Departamento de Computação - Universidade Federal do Piauí (UFPI) \\ Teresina - PI - Brasil \\ ${ }^{2}$ Centro de Informática (CIn) - Universidade Federal de Pernambuco (UFPE) \\ Recife - PE - Brasil \\ ${ }^{3}$ Departamento de Informática - Universidade Ferderal do Maranhão (UFMA) \\ São Luís - MA - Brasil \\ neclyeuxsousa@gmail.com
}

\begin{abstract}
Routing, Modulation Level and Spectrum Assignment (RMLSA) is one of the main problems studied in elastic optical networks. This work focuses on the study of guard band selection, one or more free slots between the circuits, which is used in the solutions of the RMLSA problem. In this context, a new approach, called GUARDIAN, which uses a multi layer perceptron neural network to adaptive guard band selection is proposed. Proposal performance is compared with other adaptive proposals: $A G B A$ and GBUN. The proposal achieves a reduction in the bandwidth blocking probability of at least $54.01 \%$ relative to $A G B A$ and $51.26 \%$ relative to $G B U N$.
\end{abstract}

Resumo. Routing, Modulation Level and Spectrum Assignment (RMLSA) é um dos principais problemas estudados nas redes ópticas elásticas. Este trabalho concentra-se no estudo da seleção da banda de guarda, um ou mais slots livres entre os circuitos, que é usada nas soluções do problema RMLSA. Neste contexto, uma nova abordagem, chamada de GUARDIAN, que usa uma rede neural multi layer perceptron para selecionar a banda de guarda de forma adaptativa é proposta. O desempenho da proposta é comparado com outras propostas adaptativas: AGBA e GBUN. A proposta alcança uma redução na probabilidade de bloqueio de banda de pelo menos 54,01\% em relação ao AGBA e 51,26\% em relação ao GBUN.

\section{Introdução}

A rede óptica elástica baseada na técnica de multiplexação por divisão de frequência ortogonal (OFDM - Orthogonal Frequency Division Multiplexing) foi proposta para fazer um melhor uso dos recursos ópticos para acomodar as novas aplicações da Internet cada vez maiores, tais como vídeo sob demanda e computação em nuvem [Wu et al. 2017]. Essa técnica permite a divisão do espectro óptico em pequenos intervalos de frequência chamados de slots. Dessa forma, melhora significativamente a eficiência espectral com a alocação flexível da largura de banda. Assim, cada circuito pode alocar uma quantidade diferente de slots dependendo da sua demanda de largura de banda [Chatterjee et al. 2015]. 
Utilizar de forma eficiente os recursos para garantir um melhor desempenho desse tipo de rede é um serviço que demanda a resolução de diversos problemas. Dentre os principais problemas contidos nas redes ópticas elásticas para o estabelecimento de um circuito óptico, pode-se destacar o problema de roteamento, seleção de formato de modulação e alocação de espectro (RMLSA - Routing, Modulation Level, and Spectrum Assignment) [Christodoulopoulos et al. 2011]. Para resolver tal problema é preciso executar os seguintes passos: i) definir uma rota para um par de nós origem e destino; ii) selecionar um formato de modulação adequado para essa rota e iii) escolher uma faixa de espectro livre (conjunto de slots contíguos e contínuos) na rota definida.

Um outro obstáculo nas redes ópticas elásticas é diminuir os efeitos causados pelas imperfeições de camada física na qualidade do sinal óptico. Nas redes ópticas elásticas, o sinal óptico sofre degradação à medida que se propaga pelo meio óptico devido às imperfeições da camada física. $\mathrm{O}$ acúmulo dessas degradações no sinal óptico ao longo de uma rota pode tornar a taxa de erro de bit (BER - Bit Error Rate) inaceitável no receptor, impossibilitando a comunicação [Beyranvand and Salehi 2013].

Nas redes ópticas elásticas, um ou mais slots são deixados livres entre os slots utilizados (circuitos ativos) com o objetivo de reduzir a interferência entre eles. Esses slots livres entre os circuitos são chamados de banda de guarda (GB - Guard Band) [Chatterjee et al. 2015]. A escolha do número de slots que serão usados como banda de guarda afeta diretamente a utilização dos recursos espectrais. Um valor maior de banda de guarda resultará em uma melhor qualidade de transmissão (QoT - Quality of Transmission) e também no aumento do bloqueio por ausência de recursos espectrais da rede. Por outro lado, quanto menor a banda de guarda, pior será a QoT e também ocorre a redução da probabilidade de bloqueio pela ausência de recursos espectrais da rede [Chen et al. 2017].

Dessa forma, a análise das características da rede e da requisição no momento de sua chegada devem ser levadas em consideração na definição da banda de guarda. Essas características podem ser usadas como entrada para algoritmos de machine learning como, por exemplo, o Multi Layer Perceptron (MLP), para que tais algoritmos busquem padrões para melhor selecionar o valor de banda de guarda a ser usado.

Este artigo propõe a utilização da rede MLP para determinar a banda de guarda que deverá ser utilizada para o estabelecimento de um determinado circuito. A proposta é chamada de GUARDIAN ( $\boldsymbol{G} \boldsymbol{U A R}$ d banD by multI lA yer perceptroN). A proposta consiste na utilização de um banco de dados gerado a partir da coleta de informações da rede e da banda de guarda determinada por [Monteiro et al. 2019] no momento do estabelecimento de um circuito. A nova proposta utilizando MLP é comparada com os algoritmos adaptativos apresentados em [Monteiro et al. 2019] e [Takeshita et al. 2016]. Os resultados mostram que a escolha da banda de guarda utilizando uma técnica de machine learning é mais eficiente para a redução da probabilidade de bloqueio geral da rede.

As demais seções deste artigo estão organizadas da seguinte forma. Na Seção 2 são apresentados os conceitos da arquitetura da rede óptica elástica, do problema RMLSA e do problema de seleção da banda de guarda (descrito na Subseção 2.1). A Seção 3 discute os trabalhos relacionados ao problema de seleção da banda de guarda. A Seção 4 apresenta os conceitos das redes neurais artificiais e o tipo de arquitetura usada neste tra- 
balho. O algoritmo proposto é apresentado na Seção 5. A Seção 6 apresenta um estudo de avaliação de desempenho da proposta. Por fim, as conclusões do trabalho são destacadas na Seção 7.

\section{Redes Ópticas Elásticas e o Problema da Seleção da Banda de Guarda}

O uso da tecnologia OFDM pelas redes ópticas possibilitou o surgimento de um novo tipo de rede óptica de transporte chamada de rede óptica elástica, proposta em [Jinno et al. 2009]. A tecnologia OFDM permite a segmentação do espectro óptico em uma granularidade fina, também chamada de slots de frequência. Em uma rede óptica, para que haja comunicação entre os nós, é necessário que sejam estabelecidos circuitos ópticos. Os circuitos ópticos são compostos por um ou mais slots de frequência. A quantidade de slots a ser usada para cada circuito óptico depende da largura de banda requerida. A Figura 1 apresenta um exemplo de divisão do espectro óptico em slots de frequência.

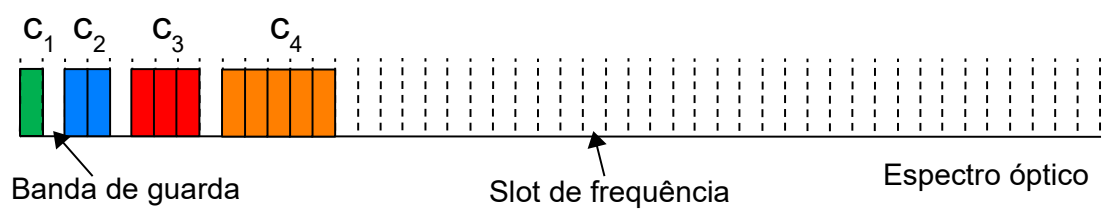

\section{Figura 1. Exemplo de divisão do espectro óptico em slots de frequência [Monteiro et al. 2019].}

Na Figura 1, os slots brancos representam slots livres e os slots coloridos representam slots ocupados por circuitos. Os slots livres entre os slots ocupados por circuitos representam a banda de guarda. A banda de guarda é usada para reduzir a interferência entre circuitos adjacentes [Zhao et al. 2015]. Ilustra-se ainda na Figura 1 a alocação de quatro circuitos com larguras de banda distintas. A alocação dos slots de frequência é ajustada para atender a largura de banda requisitada. Por exemplo, o circuito $c_{1}$ aloca apenas um slot de frequência, enquanto o circuito $c_{4}$ aloca cinco slots de frequência.

Para garantir a flexibilidade na utilização do espectro óptico, as redes ópticas elásticas utilizam duas tecnologias: Transponder com largura de banda variável (BVT - Bandwidth Variable Transponders) e comutador óptico com largura de banda variável (BV-WXC - Bandwidth Variable Wavelength Cross-Connects). Os BVTs são responsáveis por garantir a flexibilidade na utilização dos recursos espectrais na transmissão e recepção de dados nos nós de origem e destino. Os BVTs são utilizados para ajustar a largura de banda conforme a taxa de bits de transmissão ou formato de modulação adotado. Os BV-WXCs permitem a comutação óptica de circuitos que utilizam circuitos com largura de espectro ajustada dinamicamente [Chatterjee et al. 2015]. A Figura 2 ilustra a disposição dos BVTs, BV-WXCs, amplificadores e fibras ópticas em uma rede óptica elástica.

Como pode ser observado na Figura 2, os BVTs se localizam nos nós de borda da rede elástica, permitindo a transmissão e recepção dos dados dos clientes. Depois do processo de admissão de uma requisição, o circuito óptico é estabelecido a partir de um BVT no nó de origem. Este circuito atravessa de forma transparente os BV-WXC nos nós intermediários (sinais transientes), e termina em um BVT no nó de destino. 


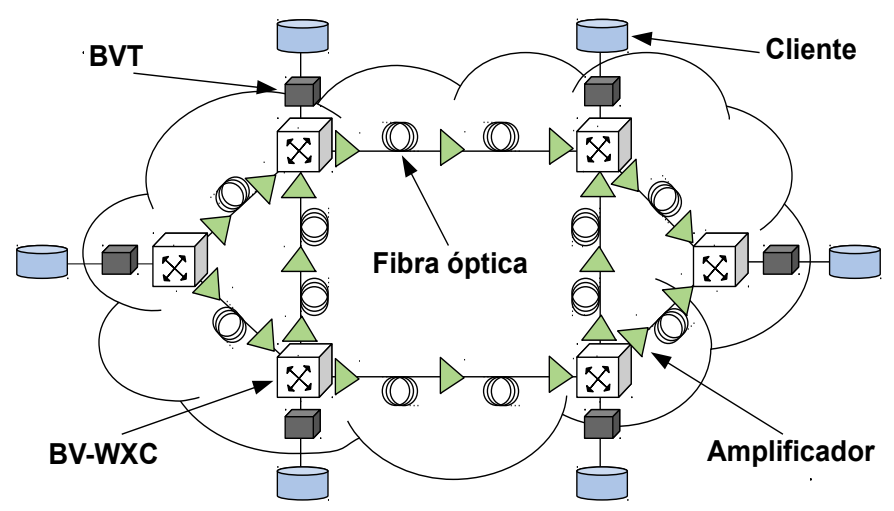

Figura 2. Arquitetura da rede óptica elástica [Monteiro et al. 2019].

Durante a transmissão do sinal óptico, quanto maior a distância percorrida na fibra, maior será a atenuação da potência de sinal. Isso ocorre de tal forma que gera uma necessidade em amplificar o sinal óptico para que este restabeleça sua potência e assim possa ser detectado no destino. Usualmente, a amplificação óptica é realizada pelo equipamento amplificador (EDFA - Erbium Doped Fiber Amplifier). No momento da amplificação, os EDFAs introduzem o ruído da emissão espontânea amplificada (ASE - Amplified Spontaneous Emission) [Saradhi and Subramaniam 2009]. Além disso, ainda ocorrem ruídos gerados pelos efeitos não lineares (NLIs - Nonlinear Impairments) quando o sinal se propaga pelas fibras.

Neste trabalho foi adotado o modelo de camada física apresentado em [Johannisson and Agrell 2014, Yan et al. 2015] para mensurar os impactos dos ruídos ASE e NLIs na relação sinal-ruído (SNR - Signal to Noise Ratio). A SNR é utilizada para a avaliação da QoT dos circuitos ópticos. Na hipótese de o valor de SNR atingido pelo circuito não ser maior ou igual a um limiar preestabelecido, o estabelecimento desse circuito pode ser bloqueado por falta de QoT. O bloqueio por falta de QoT pode ocorrer das seguintes formas: i) QoT inadequada para o novo circuito (QoTN) ou ii) QoT inadequada para um ou mais circuitos já ativos na rede (QoTO). O QoTN é o bloqueio ocorrido caso um novo circuito não alcance os níveis satisfatórios de QoT. Mesmo que um novo circuito alcance tal requisito, ainda poderá ocorrer bloqueio caso o estabelecimento do desse circuito afete na QoT dos circuitos já estabelecidos na rede, ocorrendo assim o QoTO [Fontinele et al. 2017].

Para as redes ópticas serem utilizadas de forma eficaz é necessário solucionar o problema RMLSA. A primeira etapa para resolução do problema RMLSA é escolher uma rota entre os nós de origem e destino da requisição. A Figura 3, apresenta uma topologia exemplo e mostra três possíveis escolhas de rota para uma requisição com origem no nó 1 e destino no nó 4 . As três opções possíveis de rota são: i) 1-2-3-4; ii) 1-2-5-4 e iii) $1-6-5-4$.

Após selecionar a rota, o passo seguinte é a definição do formato de modulação a ser usado. O formato de modulação escolhido e a taxa de transmissão requisitada determinam a quantidade de slots exigida para atender a requisição. Modulações com uma eficiência espectral maior (por exemplo, 32QAM - Quadrature Amplitude Modulation) tendem a sofrer mais ruídos oriundos da camada física, por essa razão possuem um al- 


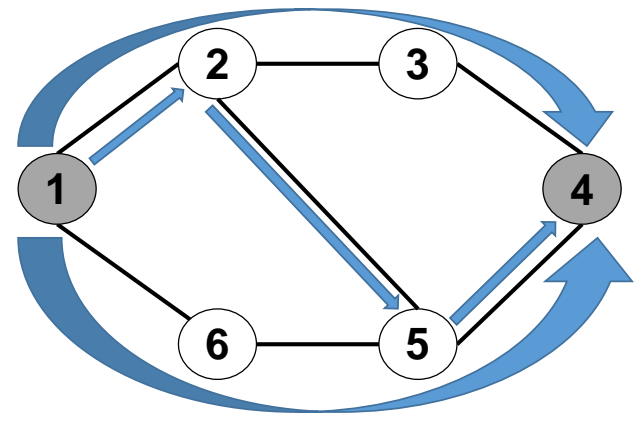

Figura 3. Topologia exemplo e problema de roteamento entre os nós 1 e 4.

cance óptico menor. Consequentemente, a seleção do formato de modulação está relacionada com o tamanho da rota escolhida. Modulação menos complexas são adequadas para rotas maiores pois alcançam uma distância maior (por exemplo, BPSK - Binary Phase Shift Keying).

Por fim, é necessário selecionar uma faixa do espectro apropriada para alocar o circuito óptico. Para isso, é preciso respeitar as restrições de continuidade e contiguidade. A restrição de continuidade determina que o sinal óptico mantenha-se no mesmo intervalo espectral em todos os enlaces da rota, ou seja, a mesma faixa de slots deve estar livre em todos os enlaces para atender a requisição. Na restrição contiguidade, requisições que exigem 2 ou mais slots, estes devem ser alocados de forma adjacente.

Conforme os circuitos vão sendo estabelecidos e suas conexões finalizadas, o espectro vai ficando fragmentado e isso aumenta a probabilidade das restrições de continuidade e contiguidade não serem atendidas. Como resultado, isso acarretaria em um número maior de bloqueios. O bloqueio por Fragmentação acontece quando a rede possui slots suficientes para atender a requisição, porém esses slots não respeitam as restrições de continuidade e contiguidade. Quando a rede não possui slots suficientes para atender a requisição, ocorre bloqueio por Ausência de Espectro Livre (AEL).

\subsection{Problema da Seleção da Banda de Guarda}

Como já relatado anteriormente, usa-se a banda de guarda com o objetivo de reduzir a interferência entre os circuitos adjacentes no espectro. Quando a faixa de slots livres que atende as restrições de continuidade e contiguidade está sendo selecionada pelo algoritmo RMLSA, ele deve também respeitar a banda de guarda entre os circuitos já ativos na rede.

O operador da rede deve ter cuidado com a quantidade de slots utilizados como banda de guarda para o estabelecimentos dos circuitos. Uma quantidade insuficiente de slots usados como banda de guarda pode impedir um circuito óptico de atingir o seu destino em consequência dos ruídos causados pela camada física. Em contrapartida, uma atribuição desnecessária pode levar a uma ineficiência espectral. Por isso, uma otimização durante a escolha da banda de guarda é importante no planejamento das redes ópticas elásticas.

\section{Trabalhos Relacionados}

Alguns trabalhos [Chen et al. 2013, Takeshita et al. 2016, Chen et al. 2017, Monteiro et al. 2019] encontrados na literatura analisam o problema de adaptar o 
tamanho da banda de guarda para realizar um trade-off entre bloqueios por QoT e bloqueios devido a falta de recursos espectrais.

Em [Chen et al. 2013] é feito um estudo de probabilidade de bloqueio com dois tipos de roteamento: singlepath e multipath. Os autores estudam também como diferentes tamanhos de banda de guarda podem afetar a performance dos algoritmos de roteamento. Contudo, em [Chen et al. 2013] os autores não utilizam uma maneira de mensurar a QoT dos circuitos e nem a interferência entre eles. Assim, um valor de banda de guarda maior leva a uma probabilidade de bloqueio maior. Isso ocorre porque todos os bloqueio são gerados devido à falta de recursos espectrais.

Os autores em [Takeshita et al. 2016] apresentam um algoritmo de alocação de banda de guarda chamado de Adaptive Guard Band Assignment (AGBA). O AGBA tem como objetivo evitar a atribuição exagerada de banda de guarda e dessa forma conseguir um uso mais eficiente do espectro óptico. O algoritmo AGBA usa apenas dois valores para atribuir a banda de guarda (1 e 2 slots). Esses valores são atribuídos de acordo com a quantidade de saltos da rota escolhida. Caso a rota escolhida para um determinado circuito possuir 4 ou menos saltos, a banda de guarda atribuída será igual a 1 slot. Caso contrário, a banda de guarda será igual a 2 slots.

Em [Chen et al. 2017] os autores realizam um estudo para identificar o tamanho de banda de guarda ideal com o objetivo de minimizar os impactos causados por efeitos não lineares. Os autores mostram um estudo onde à medida que a banda de guarda aumenta, o bloqueio por QoT diminui rapidamente. No entanto, o bloqueio por falta de recursos disponíveis aumenta. O bloqueio total da rede é minimizado com uma banda de guarda de $7 \mathrm{GHz}$ ou $8 \mathrm{GHz}$, para o cenário estudado.

Em [Monteiro et al. 2019] os autores propõem uma nova abordagem adaptativa para a escolha da banda de guarda baseada no nível de utilização da rede. O algoritmo é chamado de Guard Band according to Use of the Network (GBUN). O algoritmo GBUN busca selecionar um valor de banda de guarda menor quando a rede se encontra mais utilizada e um valor de banda de guarda maior quando a rede se encontra pouco utilizada. A proposta é comparada com a proposta apresentada em [Takeshita et al. 2016] e alguns valores fixos para a banda de guarda encontrados na literatura.

A Tabela 1 mostra uma ligeira comparação entre as propostas supracitadas. A comparação entre as propostas leva em consideração os seguintes aspectos: i) A proposta considera os efeitos causados pela camada física? ii) A proposta atribui banda de guarda dinamicamente? iii) Quantos valores são atribuídos a banda de guarda? iv) Qual o número de parâmetros avaliados antes de atribuir um valor para a banda de guarda?

Tabela 1. Comparativo entre as propostas de definição de banda de guarda encontradas na literatura.

\begin{tabular}{c|c|c|c|c}
\hline Trabalho & i & ii & iii & iv \\
\hline [Chen et al. 2013] & Não & Não & 3 & 0 \\
\hline [Takeshita et al. 2016] & Sim & Sim & 2 & 1 \\
\hline [Chen et al. 2017] & Sim & Não & 14 & 0 \\
\hline [Monteiro et al. 2019] & Sim & Sim & 8 & 1 \\
\hline
\end{tabular}

As propostas listadas na Tabela 1 não exploram técnicas computacionais mais 
eficientes ou que possuem uma capacidade maior de otimização como as de machine learning. Este trabalho propõe o uso de uma dessas técnicas para resolver o problema de escolha da banda de guarda. A técnica de machine learning utilizada na proposta é apresentada na Seção 4. O algoritmo proposto é apresentado na Seção 5.

\section{Redes Neurais Multi Layer Perceptron}

Redes neurais artificiais são modelos computacionais inspirados no comportamento do cérebro humano, que possuem uma alta capacidade de reconhecimento de padrões [Borges et al. 2016]. Neste trabalho foi utilizado as redes neurais de camadas múltiplas ou Multi Layer Perceptrons (MLPs). As principais características das MLPs que atraem os pesquisadores são sua capacidade de autoaprendizagem, sem necessidade de conhecer a relação de dados, capacidade de ajuste e aplicabilidade para modelar vários problemas [Khokhar et al. 2015].

A MLP consiste de um sistema de neurônios interconectados, que representa um mapeamento não linear entre um vetor de entrada e um vetor de saída [Gardner and Dorling 1998]. Os neurônios são conectados por pesos e sinais de saídas, que é uma função da soma ponderada da entrada pelo peso por uma função simples de transferência ou ativação não linear. A saída de um neurônio é usada como entrada para os próximos neurônios da rede, isso implica em uma direção de processamento, assim, a rede MLP é conhecida como uma rede neural feed-foward. Uma MLP pode ter uma ou mais camadas ocultas e, finalmente, uma camada de saída. A MLP é descrita como uma rede totalmente conectada, ou seja, cada neurônio é conectado a cada neurônio da camada seguinte.

Uma rede MLP tem a capacidade de aprender por meio de treinamento. O treinamento requer um conjunto de dados que consiste em atributos, que serão usados como entrada, e uma classe objetivo associada, que é a saída desejada. A Figura 4 mostra o processo para extração dados para montar a base de dados e o treinamento da rede.

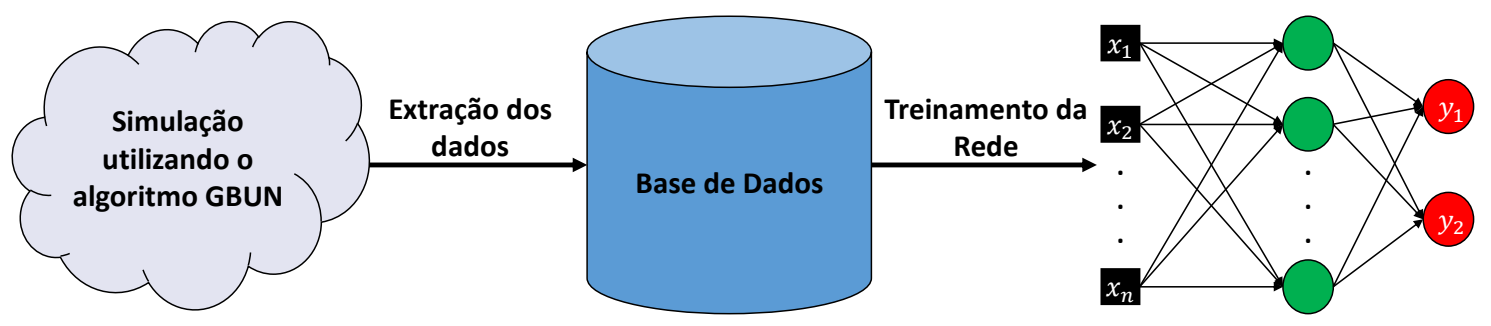

Figura 4. Fluxograma de extração dos dados e treinamento da Rede Neural.

A MLP aprende de maneira supervisionada, ou seja, durante o treinamento, os registros da base de dados (entrada e saída) são apresentadas repetidamente para a rede MLP, e os pesos são ajustados até que o mapeamento entre as entradas e saídas associadas ocorram. Durante o treinamento, as saídas podem não corresponder com as saídas desejadas, dessa forma um sinal de erro é definido como a diferença entre a saída desejada e a real. A rede utiliza esse erro para determinar o grau de ajuste dos pesos a fim de diminuir o erro geral das camadas da rede MLP. Existem muitos algoritmos para o treinamento da MLP dentre eles o backprogation [Hecht-Nielsen 1992], que realiza o cálculo do gradiente local do erro e altera os pesos na direção do gradiente local mais acentuado, assim espera-se que os pesos convirjam para o mínimo global. 
No processo de treinamento da MLP várias decisões precisam ser tomadas em relação as configurações da rede, a fim de obter um melhor desempenho. Quantidade de épocas, de neurônios, de camadas ocultas, valor da taxa de aprendizagem e etc são exemplos de parâmetros que precisam ser otimizados. Ao se treinar uma MLP, geralmente se está interessado em descobrir o melhor valor para esses parâmetros, objetivando conseguir um melhor desempenho da rede. No entanto, por conta da quantidade de parâmetros que precisam ser otimizados, as redes MLP são propensas a vários problemas como overfitting [Hawkins 2004] e oscilações no ótimo [Wen Jin et al. 2000]. Uma das técnicas que podem ser utilizadas para evitar o overfitting é o Early stop (parada precoce), que permite especificar um grande número arbitrário de épocas de treino e parar o treino quando o desempenho do modelo deixa de melhorar num conjunto de dados. Para evitar oscilações no ótimo, pode-se utilizar, durante o treinamento, taxa de aprendizagem dinâmica. Dessa forma o valor da taxa de aprendizagem pode ser diminuída a medida em que a rede não apresenta melhora, a fim de se aproximar do valor ótimo evitando oscilações.

Nesse contexto, a rede MLP utilizada neste trabalho possui 4 camadas completamente conectadas. Cada camada possui respectivamente, 128, 64, 32 e 8 neurônios utilizando a função de ativação ReLU (Rectified Linear Units) [LeCun et al. 2015] nas três primeiras camadas e a função softmax, que fornece o valor da probabilidade do dado de entrada pertencer a uma classe correspondente [Wang and Chen 2019].

\section{Guard Band by Multi Layer Perceptron - GUARDIAN}

O algoritmo proposto neste trabalho para seleção da banda de guarda adaptativa utiliza a técnica descrita na Seção 4. O algoritmo é chamado de GUARDIAN (GUARd banD by multI lAyer perceptroN). O algoritmo adaptativo proposto em [Monteiro et al. 2019] é aplicado para gerar a base de dados utilizada durante a fase de treinamento da rede, conforme Figura 4. A base de dados contém 882 mil registros. Cada registro é composto por características da rede e da requisição no momento em que o circuito foi estabelecido durante a simulação.

As características extraídas são as seguintes: i) total de slots usados pelo enlace mais utilizado da rota encolhida, ii) utilização desse enlace, iii) número de circuitos ativos no enlace, iv) número de enlaces da rota, v) total de slots livres na rota, vi) utilização geral da rede, vii) modulação escolhida e viii) SNR do circuito. Estas por sua vez são as variáveis de entrada que são passadas para a rede MLP. É armazenado também na base de dados o valor da banda de guarda escolhida, que por sua vez é a variável de saída da rede MLP. A Tabela 2 apresenta um exemplo de alguns registros contidos na base de dados extraída para treinamento a partir do algoritmo GBUN na topologia Cost239.

Tabela 2. Exemplo de alguns registros da base de dados.

\begin{tabular}{c|c|c|c|c|c|c|c|c}
\hline i & ii & iii & iv & iv & vi & vii & viii & Banda de Guarda \\
\hline 4 & 0,0125 & 2 & 3 & 950 & 0,0023 & 8QAM & 13,59272 & 8 \\
\hline 105 & 0,3281 & 34 & 1 & 215 & 0,2114 & 16QAM & 17,23032 & 2 \\
\hline 32 & 0,1 & 10 & 2 & 577 & 0,0916 & 16QAM & 16,51364 & 6 \\
\hline 87 & 0,2718 & 29 & 1 & 233 & 0,2387 & 32QAM & 19,19441 & 1 \\
\hline
\end{tabular}

Além disso, as instâncias que apresentam algum tipo de bloqueio são removidas durante a fase de extração dos dados para que a MLP possa aprender os padrões no qual 
a banda de guarda é alocada sem que haja bloqueio. A banda de guarda pode assumir 8 valores (1 a 8 slots), os mesmos valores usados por [Monteiro et al. 2019]. A Figura 5 abaixo apresenta o fluxograma para aplicação da proposta desenvolvida neste trabalho.

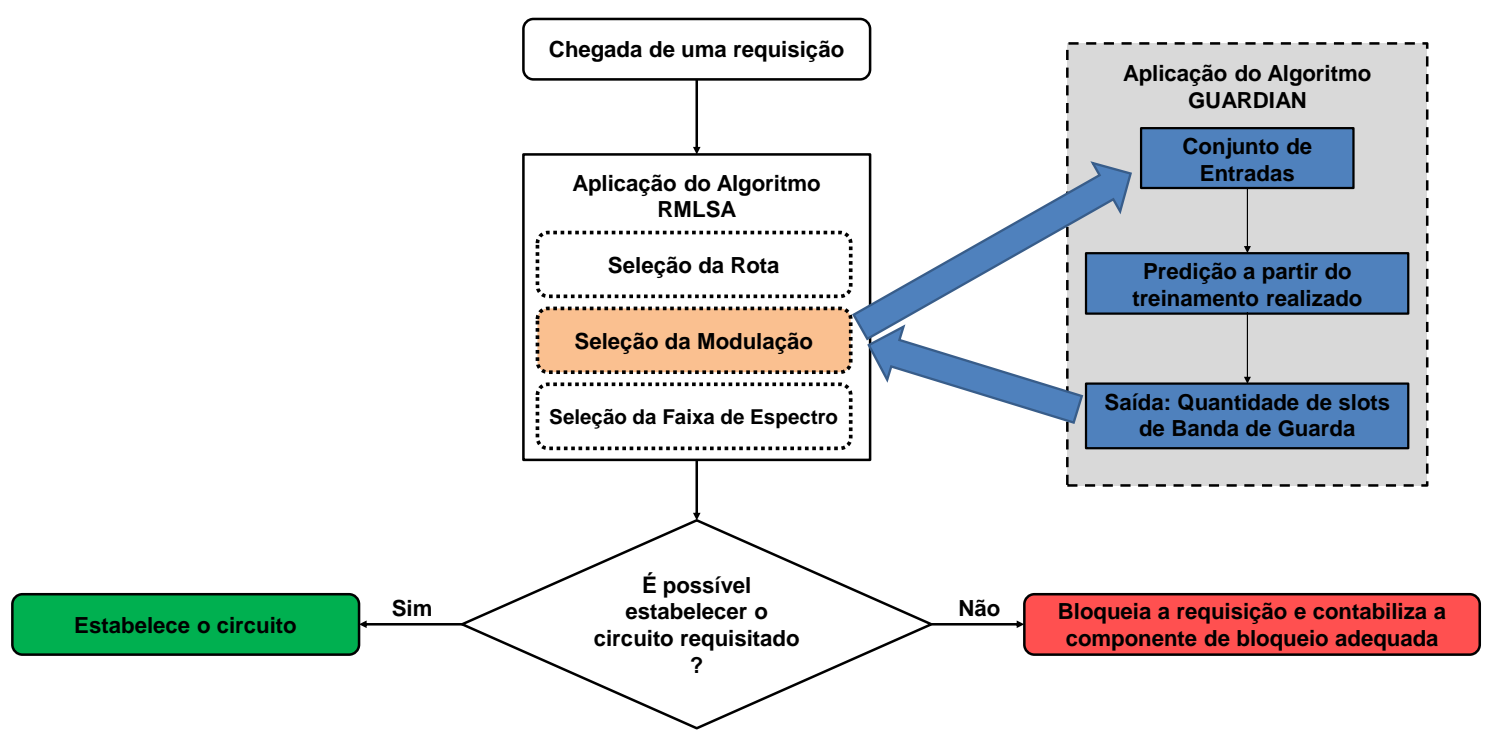

Figura 5. Fluxograma de aplicação do algoritmo GUARDIAN.

Conforme pode ser observado na Figura 5, após a chegada de uma requisição, é feito a escolha da rota e da modulação pelo algoritmo RMLSA. Durante essa etapa, é aplicado o algoritmo proposto neste trabalho. Um conjunto de dados com as características extraídas, listadas acima, são passadas como entrada para a rede MLP treinada. A rede MLP então realiza a predição do valor de banda de guarda a partir dos novos dados de entrada e do treinamento realizado anteriormente. No final, o algoritmo RMLSA seleciona uma faixa do espectro apropriada para alocar o circuito óptico.

Por fim, o plano de controle verifica se é possível realizar o estabelecimento do novo circuito requisitado. Se não for possível o estabelecimento do novo circuito é identificado e contabilizado o bloqueio por uma das componentes de bloqueio já mencionadas (Fragmentação, AEL, QoTN ou QoTO). Caso seja possível o estabelecimento do novo circuito, o plano de controle alocará os recursos definidos anteriormente pelo algoritmo RMLSA, incluindo a banda de guarda definida pelo algoritmo GUARDIAN.

O algoritmo empregado para resolução do problema RMLSA neste trabalho foi o Complete Sharing [Wang and Mukherjee 2014]. O algoritmo Complete Sharing é um algoritmo RMLSA integrado que foi proposto para lidar com a fragmentação do espectro. Ele utiliza roteamento com as $k$ rotas candidatas e seleciona a rota que possibilita alocar a faixa de slots mais próxima do início do espectro. O Complete Sharing utiliza o First Fit para a tarefa de alocação de espectro. Para a seleção de modulação é utilizado o algoritmo de seleção do formato de modulação pela QoT [Fontinele et al. 2017].

\section{Avaliação de Desempenho}

A implementação da rede MLP foi feita por meio do Tensorflow [Abadi et al. 2016] e Keras [Chollet et al. 2015]. A avaliação de desempenho da rede MLP no experimento é verificada por meio da acurácia. Para o cálculo da acurácia foi utilizada a matriz de 
confusão e a partir dela pode-se calcular o VP (Verdadeiro Positivo), FP (Falso Positivo), VN (Verdadeiro Negativo) e FN (Falso Negativo) [Sokolova and Lapalme 2009]. A acurácia é obtida pelo número de VPs mais VNs divididos pelo número total de amostras no conjunto de teste.

O treinamento da rede MLP consiste de dois estágios usando o otimizador Adam [Kingma and Ba 2014], taxa de aprendizagem dinâmica e Early-Stop [Wang and Chen 2019]. No primeiro estágio a taxa de aprendizado é igual a 0,001 e o treinamento segue para o próximo passo quando o valor de perca piora por 10 vezes consecutivas. No segundo estágio a taxa de aprendizagem é decrescida para 0,0001 e o treinamento finaliza quando o valor de perda piora por 20 vezes consecutivas. Os valores da taxa de treinamento e quantidade consecutiva de piora do valor de perda, para cada estágio, é definido de forma empírica por meio da análise do treinamento. Nessa etapa, toda a base de dados foi utilizada com o objetivo de obter uma maior quantidade e diversidade de amostras.

O problema é formulado com um problema de classificação, dessa forma a acurácia é utilizada para avaliar o treinamento da rede. A rede MLP obteve uma taxa de acurácia satisfatória, acima de $96 \%$ para ambos os cenários estudados, atingindo 96,79\% e $98,57 \%$ para as topologias Cost239 e NSFNet, respectivamente. Isso demostra a viabilidade de obter o valor da banda de guarda por meio da análise das características da rede e da requisição por meio da rede MLP.

A proposta deste trabalho, GUARDIAN, foi comparada com os algoritmos adaptativos AGBA e GBUN. O algoritmo Complete Sharing foi empregado para resolver o problema RMLSA com $k$ igual a 3. Como forma de avaliação, foi considerada a métrica probabilidade de bloqueio de banda e também foram analisadas as componentes da probabilidade de bloqueio de banda. As componentes avaliadas foram: Fragmentação, QoTN (QoT inaceitável para um novo circuito), QoTO (QoT inaceitável para os circuitos já ativos na rede) e AEL (Ausência de Espectro Livre).

As simulações foram realizadas usando o simulador SNetS (SLICE Network Simulator) [Fontinele et al. 2017]. Em cada simulação foram geradas 100.000 requisições de circuitos ópticos com sete níveis de granularidade: 100, 150, 200, 250, 300, 350 e 400 Gbps, com as proporções 7:6:5:4:3:2:1, respectivamente. A geração de requisições segue a distribuição de Poisson com a carga de tráfego distribuída uniformemente entre todos os pares de nós origem e destino. Para cada simulação foram realizadas dez replicações com diferentes sementes de geração de variável aleatória. Todos os resultados possuem nível de confiança de $95 \%$.

As topologias consideradas nas simulações são a Cost239 e a NSFNet (National Science Foundation Network) (Figura 6). Foram considerados 5 formatos de modulações neste trabalho: BPSK, 4QAM, 8QAM, 16QAM e 32QAM e os seus respectivos limiares de SNR por símbolo são 5,5, 8,5, 12,5, 15,1 e 18,1 dB [Ives et al. 2014]. Todos os enlaces da rede são bidirecionais e possuem largura de banda do espectro dividida em 320 slots de frequência. Um slot de frequência possui largura de banda de 12,5 GHz. Os ganhos dos amplificadores são ajustados para compensar as perdas dos dispositivos (comutadores, multiplexadores e demultiplexadores) e das fibras. Outros parâmetros utilizados nas simulações estão listados na Tabela 3. 


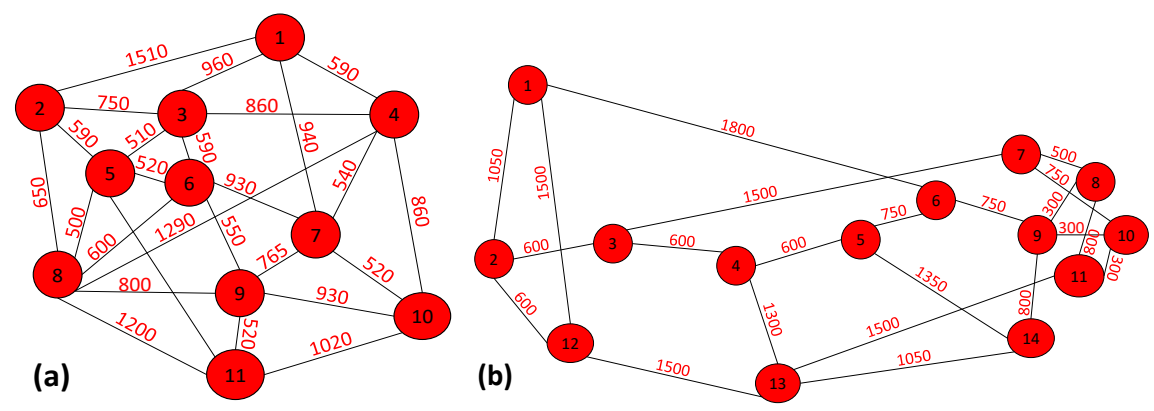

Figura 6. Topologias utilizadas: (a) Cost239 e (b) NSFNet

Tabela 3. Parâmetros de camada física utilizados nas simulações.

\begin{tabular}{c|c}
\hline Descrição & Valor \\
\hline Densidade espectral de potência do sinal & $-23 \mathrm{dBm} / \mathrm{GHz}$ \\
Atenuação da fibra $(\alpha)$ & $0,2 \mathrm{~dB} / \mathrm{km}$ \\
Parâmetro de dispersão da fibra $(D)$ & $16 \mathrm{ps} /(\mathrm{nm} \cdot \mathrm{km})$ \\
Coeficiente não linear da fibra $(\gamma)$ & $1,3(\mathrm{Wkm})^{-1}$ \\
Tamanho de um span $\left(L_{s}\right)$ & $80 \mathrm{~km}$ \\
Figura de ruído do amplificador $(N F)$ & $5 \mathrm{~dB}$ \\
\hline
\end{tabular}

A Figura 7 apresenta a probabilidade de bloqueio de banda obtida pelos algoritmos avaliados neste trabalho em função da carga da rede para as topologias Cost239 e NSFNet. O algoritmo GUARDIAN alcançou o melhor desempenho em termos de probabilidade de bloqueio de banda para todos os pontos de carga analisados na topologia Cost239 (Figura 7(a)). Considerando o ponto de mais alta carga (750 Erlangs) na topologia Cost239, o algoritmo GUARDIAN reduziu a probabilidade de bloqueio geral em aproximadamente $73,56 \%$ e 71,22\% em relação as algoritmos AGBA e GBUN, respectivamente.

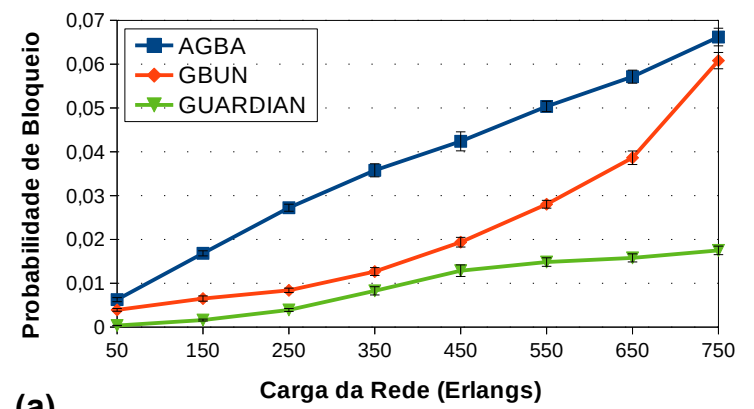

(a)

Figura 7. Probabilidade de bloqueio de banda para as topologias (a) Cost239 e (b) NSFNet.

Na topologia NSFNet (Figura 7(b)), o algoritmo GUARDIAN também alcançou o melhor desempenho em termos de probabilidade de bloqueio de banda para todos os pontos de carga analisados. Considerando a carga de 400 Erlangs na topologia NSFNet, o algoritmo GUARDIAN reduziu a probabilidade de bloqueio geral em aproximadamente $54,01 \%$ e 51,26\% em relação aos algoritmos AGBA e GBUN, respectivamente.

As Tabelas 4 e 5 exibem as componentes da probabilidade de bloqueio de banda 
obtidas com os algoritmos apresentados na Figura 7. Os resultados apresentados nas Tabelas 4 e 5 foram obtidos no último ponto de carga analisado em cada topologia. Carga de 750 Erlangs para a topologia Cost239 e carga de 400 Erlangs para a topologia NSFNet.

\section{Tabela 4. Componentes da probabilidade de bloqueio de banda para a topologia Cost239 na carga de 750 Erlangs.}

\begin{tabular}{c|c|c|c|c}
\hline Algoritmo & Fragmentação & QoTN & QoTO & AEL \\
\hline AGBA & $0 \%$ & $0 \%$ & $100 \%$ & $0 \%$ \\
\hline GBUN & $0 \%$ & $0 \%$ & $100 \%$ & $0 \%$ \\
\hline GUARDIAN & $0 \%$ & $0 \%$ & $100 \%$ & $0 \%$ \\
\hline
\end{tabular}

\section{Tabela 5. Componentes da probabilidade de bloqueio de banda para a topologia} NSFNet na carga de 400 Erlangs.

\begin{tabular}{c|c|c|c|c}
\hline Algoritmo & Fragmentação & QoTN & QoTO & AEL \\
\hline AGBA & $0,27 \%$ & $0 \%$ & $99,70 \%$ & $0,03 \%$ \\
\hline GBUN & $0,95 \%$ & $0 \%$ & $98,98 \%$ & $0,07 \%$ \\
\hline GUARDIAN & $0 \%$ & $0 \%$ & $97,13 \%$ & $2,87 \%$ \\
\hline
\end{tabular}

Observa-se pelas Tabelas 4 e 5 que a componente que mais impactou no bloqueio geral para todos os algoritmos em ambas topologias foi a QoTO. Vale destacar que a utilização do algoritmo Complete Sharing contribui para a redução dos bloqueios gerados pelas componentes Fragmentação, AEL e QoTN.

O melhor desempenho do algoritmo proposto está relacionado diretamente com a capacidade de reconhecimento de padrões da rede MLP. Através do banco de dados a rede MLP consegue inferir, por meio das características extraídas da rede e da requisição, a banda de guarda de forma mais eficiente, reduzindo o bloqueio geral da rede.

\section{Conclusão}

Este artigo propõe o algoritmo GUARDIAN que realiza a seleção da banda de guarda utilizando uma rede neural multi layer perceptron. O uso dessa técnica permite que o GUARDIAN aprenda a inferir a banda de guarda ideal para cada requisição de circuito.

Uma avaliação de desempenho foi realizada comparando o algoritmo GUARDIAN com outras propostas adaptativas encontradas na literatura: AGBA e GBUN. O estudo foi realizado com as topologias Cost239 e NSFNet. Os resultados mostraram que o algoritmo GUARDIAN alcançou o melhor desempenho em termos de probabilidade de bloqueio de banda em comparação aos algoritmos AGBA e GBUN.

Como trabalho futuro pretende-se explorar mais técnicas de machine learning. Por meio dessas técnicas pode-se melhorar a seleção da banda de guarda e também descobrir os padrões associados a escolha feita pelos algoritmos para a seleção da banda de guarda. Um dos algoritmos que pode ser explorado é a rede convolucional, que realiza a extração de características de forma automatizada. Dessa forma, pode-se utilizar a rede convolucional para extrair as características a partir do estado atual da rede. Além disso, por meio da análise do banco de dados gerado por essas técnicas pode-se inferir, através da árvore de decisão, o conhecimento adquirido por essas técnicas. 


\section{Referências}

Abadi, M., Barham, P., Chen, J., Chen, Z., Davis, A., Dean, J., Devin, M., Ghemawat, S., Irving, G., Isard, M., et al. (2016). Tensorflow: a system for large-scale machine learning. In $O S D I$, volume 16, pages 265-283.

Beyranvand, H. and Salehi, J. (2013). A quality-of-transmission aware dynamic routing and spectrum assignment scheme for future elastic optical networks. Journal of Lightwave Technology, 31(18):3043-3054.

Borges, F. A., Fernandes, R. A., Silva, I. N., and Silva, C. B. (2016). Feature extraction and power quality disturbances classification using smart meters signals. IEEE Transactions on Industrial Informatics, 12(2):824-833.

Chatterjee, B. C., Sarma, N., and Oki, E. (2015). Routing and spectrum allocation in elastic optical networks: A tutorial. IEEE Communications Surveys \& Tutorials, 17(3):1776-1800.

Chen, C., Ju, M., Xiao, S., Zhou, F., and Yang, X. (2017). Minimizing total blocking by setting optimal guard band in nonlinear elastic optical networks. In 2017 19th International Conference on Transparent Optical Networks (ICTON), pages 1-4.

Chen, X., Zhong, Y., and Jukan, A. (2013). Multipath routing in elastic optical networks with distance-adaptive modulation formats. In 2013 IEEE International Conference on Communications (ICC), pages 3915-3920.

Chollet, F. et al. (2015). Keras: Deep learning library for theano and tensorflow. URL: https://keras. io/k, 7(8).

Christodoulopoulos, K., Tomkos, I., and Varvarigos, E. (2011). Elastic bandwidth allocation in flexible OFDM-based optical networks. Journal of Lightwave Technology, 29(9):1354-1366.

Fontinele, A., Santos, I., Neto, J. N., Campelo, D. R., and Soares, A. (2017). An efficient IA-RMLSA algorithm for transparent elastic optical networks. Computer Networks, 118(Supplement C): 1 - 14.

Gardner, M. W. and Dorling, S. (1998). Artificial neural networks (the multilayer perceptron)-a review of applications in the atmospheric sciences. Atmospheric environment, 32(14-15):2627-2636.

Hawkins, D. M. (2004). The problem of overfitting. Journal of chemical information and computer sciences, 44(1):1-12.

Hecht-Nielsen, R. (1992). Theory of the backpropagation neural network. In Neural networks for perception, pages 65-93. Elsevier.

Ives, D. J., Bayvel, P., and Savory, S. J. (2014). Physical layer transmitter and routing optimization to maximize the traffic throughput of a nonlinear optical mesh network. In 2014 International Conference on Optical Network Design and Modeling, pages 168-173.

Jinno, M., Takara, H., Kozicki, B., Tsukishima, Y., Sone, Y., and Matsuoka, S. (2009). Spectrum-efficient and scalable elastic optical path network: architecture, benefits, and enabling technologies. IEEE Communications Magazine, 47(11):66-73. 
Johannisson, P. and Agrell, E. (2014). Modeling of nonlinear signal distortion in fiberoptic networks. Journal of Lightwave Technology, 32(23):4544-4552.

Khokhar, S., Zin, A. A. B. M., Mokhtar, A. S. B., and Pesaran, M. (2015). A comprehensive overview on signal processing and artificial intelligence techniques applications in classification of power quality disturbances. Renewable and Sustainable Energy Reviews, 51:1650-1663.

Kingma, D. P. and Ba, J. (2014). Adam: A method for stochastic optimization. arXiv preprint arXiv:1412.6980.

LeCun, Y., Bengio, Y., and Hinton, G. (2015). Deep learning. nature, 521(7553):436.

Monteiro, N., Fontinele, A., Santos, I. G., Oliveira, A., Campelo, D., and Soares, A. (2019). Novo algoritmo para provisão de banda de guarda adaptativa em redes Ópticas elásticas. In Anais do XXXVII Simpósio Brasileiro de Redes de Computadores e Sistemas Distribuídos, pages 307-320, Gramado, RS, Brasil. SBC.

Saradhi, C. V. and Subramaniam, S. (2009). Physical layer impairment aware routing (PLIAR) in WDM optical networks: Issues and challenges. Commun. Surveys Tuts., 11(4):109-130.

Sokolova, M. and Lapalme, G. (2009). A systematic analysis of performance measures for classification tasks. Information Processing \& Management, 45(4):427 - 437.

Takeshita, H., Noguchi, H., Abe, J., Fujisawa, S., and Tajima, A. (2016). Adaptive guardband assignment with adaptive spectral profile equalizer to improve spectral usage of impairment-aware elastic optical network. In ECOC 2016; 42nd European Conference on Optical Communication, pages 1-3.

Wang, R. and Mukherjee, B. (2014). Spectrum management in heterogeneous bandwidth optical networks. Optical Switching and Networking, 11, Part A:83 - 91.

Wang, S. and Chen, H. (2019). A novel deep learning method for the classification of power quality disturbances using deep convolutional neural network. Applied Energy, 235:1126-1140.

Wen Jin, Zhao Jia Li, Luo Si Wei, and Han Zhen (2000). The improvements of bp neural network learning algorithm. In WCC 2000 - ICSP 2000. 2000 5th International Conference on Signal Processing Proceedings. 16th World Computer Congress 2000, volume 3, pages 1647-1649 vol.3.

Wu, J., Ning, Z., and Guo, L. (2017). Energy-efficient survivable grooming in softwaredefined elastic optical networks. IEEE Access, 5:6454-6463.

Yan, L., Agrell, E., Wymeersch, H., Johannisson, P., Di Taranto, R., and Brandt-Pearce, M. (2015). Link-level resource allocation for flexible-grid nonlinear fiber-optic communication systems. IEEE Photonics Technology Letters, 27(12):1250-1253.

Zhao, J., Wymeersch, H., and Agrell, E. (2015). Nonlinear impairment aware resource allocation in elastic optical networks. In 2015 Optical Fiber Communications Conference and Exhibition (OFC), pages 1-3. 